\title{
A Novel Concept of Uncertainty Optimization Based Multi-Granular Rough Set and Its Application
}

\author{
Pradeep Shende*, Arvind Kumar Sinha \\ Department of Mathematics, National Institute of Technology Raipur, Chhattisgarh, India
}

Received May 3, 2021; Revised June 17, 2021; Accepted July 19, 2021

Cite This Paper in the following Citation Styles

(a): [1] Pradeep Shende, Arvind Kumar Sinha, ”A Novel Concept of Uncertainty Optimization Based Multi-Granular Rough Set And Its Application," Mathematics and Statistics, Vol.9, No.4, pp. 608-616, 2021. DOI: 10.13189/ms.2021.090420

(b): Pradeep Shende, Arvind Kumar Sinha, (2021). A Novel Concept of Uncertainty Optimization Based Multi-Granular Rough Set And Its Application. Mathematics and Statistics, 9(4), 608-616. DOI: 10.13189/ms.2021.090420

Copyright $\odot 2021$ by authors, all rights reserved. Authors agree that this article remains permanently open access under the terms of the Creative Commons Attribution License 4.0 International License

\begin{abstract}
Data is generating at an exponential pace with the advancement in information technology. Such data highly contain uncertain and vague information. The rough set approximation is a way to find information in the data-set under uncertainty and to classify objects of the dataset. This work presents a mathematical approach to evaluate the data-sets uncertainties and their application to data reduction. In this work, we have extended the multi-granulation variable precision rough set in the context of uncertainty optimization. We develop an uncertainty optimization-based multi-granular rough set (UOMGRS) to minimize the uncertainties in the data set more effectively. Using UOMGRS, we find the most informative attribute in the feature space. It is desirable to minimize the rough set boundary region using the attribute having the highest approximation quality. Thus we group the attributes whose relative quality of approximation is the maximum to maximize the positive region and to minimize the uncertain region. We compare the UOMGRS with the single granulation rough set (SGRS) and the multi-granular rough set (MGRS). By our proposed method, we require only an average of $62 \%$ attributes for approximation whereas, SGRS and MGRS need an average of at least $72 \%$ of attributes in the data set for approximation of the concepts in the data-set. Our proposed method requires less amount of data for the classification of objects in the dataset. The method helps minimize the uncertainties in the dataset in a more efficient way.
\end{abstract}

Keywords Uncertainty Optimization, Multi-granulation, Rough Set, Optimization Algorithm, Data Reduction

\section{Introduction}

Each object possesses the information, and we can classify objects into distinct categories using this information. The object information stored using different attributes is termed a knowledge system. The attributes or features are the granules of information, and we can label object's using them. Each label in the knowledge system is termed a concept in the knowledge system [1]. The rough set theory (RST) proposed by Pawlak [2] is a practical approach for approximation of sets in the knowledge system. It provides many useful tools for data analysis using the information available in the knowledge system. The growing applications of RST have developed a great deal of interest among researchers of expert systems, and artificial intelligence. These includes, but not limited to data engineering, knowledge acquisition, decision making $[3,4,5,6,7]$. RST is very advantageous as it handles data at hand and requires no posterior information [8]. One of the applications of RST is the classification of objects of the universe into different categories. The subsets of the feature set which keeps the classification of the objects intact and preserve the information of the dataset as a whole are termed as reducts of the feature space [2]. Rough set reducts for feature selection is the wrapper method in which feature subsets are formed using their quality of approximation of the object classification. Quality of approximation is used for classification of objects into distinct categories. The new possibilities of application of artificial intelligence methods for high-precision solution of boundary value problems is studied in [9]. Reducts are mainly used for feature selection and data reduction. RST uses a single binary relation for defining the set approximations. The indiscernibility relation is an equivalence relation that partition the universal set into equivalence classes. The classes of this indiscernibility relation described the objects-classification as specific classification and possible classification. This concept 
approximation approach is very rigid as there is no space provided for any misclassification of the objects. The exploratory spatial data analysis is studied in [10]. In a practical situation, we have to deal with uncertain information, and the main limitation of RST is that it is incapable of modelling uncertain information [8]. The variable precision rough set (VPRS) model by Ziarko is the generalization of the classical RST in which a specified degree of misclassification error is allowed in the set approximations. The main advantage of VPRS is that it minimizes the boundary region of the rough set. The VPRS model applications in data mining show its usefulness in data mining under uncertainty $[11,12,13,14]$. The classical rough set uses a single binary relation in the definition of set approximations. The multi-granulation rough set (MGRS) is the extension of the classical rough set using multi-equivalence relation on the universe of discourse. Qian et al. [15] proposed the MGRS approach in the case of multi-granulation. The MGRS model under the variable precision rough set environment was proposed by Wei et al. [16]. A specified value of misclassification error is used to find the classes of the multi-equivalence relation over the universe. The main limitation of this model is as follows:

- There is no method described for obtaining the value of misclassification error.

- The same value of misclassification error is used in the multi-equivalence relation.

In this work, we consider the value of misclassification error depending on the class inclusion values of the multiequivalence relation. We formulate an uncertainty optimization problem for minimizing the uncertainty using the multiequivalence relation over the attribute sets. We define the set approximation for variable precision multi-granulation rough set from the perspective of uncertainty optimization. The reducts obtained are used for data reduction and feature selection. We see the attribute with the highest quality of the approximation, as it is the most informative attribute in the feature space. It is desirable to minimize the uncertain region of this attribute using the positive region of the remaining attributes. Hence we group the attributes whose relative quality of approximation is the maximum for data reduction. This study's main contribution is the extension of the variable precision multigranulation rough set model from an uncertainty minimization point of view. This work gives useful results and properties in terms of multi-equivalence relation and class inclusion values. The application of the UOMGRS is discussed to find reducts of the feature space using the uncertainty optimization problem.

\section{Preliminaries}

\subsection{Pawlak's single granulation rough set ap- proximations (SGRS)}

Let $\mathbb{U}$ denotes the non-empty finite set of objects also called as universe of discourse, and Atr be the non-empty set of condition attributes, and $\{D\}$ be the decision attribute. For any $A \in A \operatorname{tr}$ and $x \in \mathbb{U}$, let $A(x)$ be the attribute value of $A$ for $x$, and $V$ be the set of all attribute values, then $(\mathbb{U}, A t r \cup\{D\}, V)$ is the representation of knowledge system. Let $x, y \in \mathbb{U}$, then $\theta_{\text {Atr }}$ be the relation defined as follows:

$$
x \theta_{\text {Atr }} y \text { iff } A(x)=A(y), \forall A \in A t r
$$

It is clear that the relation $\theta_{A t r}$ is an equivalence relation. Let the class of relation $\theta_{A t r}$ containing $x$ is denoted as $\widehat{\operatorname{Atr}}(x)$, then we have

$$
\widehat{\operatorname{Atr}}(x)=\{y \in \mathbb{U}: A(x)=A(y), \forall A \in A t r\}
$$

Let $S \subseteq \mathbb{U}$, and $x \in S$, we define the class of $x$ given the set $S$ by the relation $\theta_{A t r}$ as

$$
\widehat{\operatorname{Atr}}(x \mid S)=\{y \in S: A(x)=A(y), \quad \forall A \in A t r\}
$$

Clearly $\widehat{\operatorname{Atr}}(x \mid S) \subseteq \widehat{\operatorname{Atr}}(x)$. Ziarko [8] define the misclassification-error of the class $\widehat{A t r}(x) \in \widehat{A t r}$ in the concept $\chi$ as

$$
e(\widehat{\operatorname{Atr}}(x), \chi)=1-\frac{|\widehat{A t r}(x) \cap \chi|}{|\widehat{A t r}(x)|}
$$

where, $|X|$ is the cardinality of $X$. For any $S \subseteq \mathbb{U}$, we define the majority inclusion-relation over the set $S$ as follows:

$$
\widehat{\operatorname{Atr}}(x) \stackrel{\alpha}{\subseteq} \chi \quad \text { if and only if } e(\widehat{\operatorname{Atr}}(x \mid S), \chi) \leq \alpha
$$

For the value of $\alpha=0$, we have $\widehat{\operatorname{Atr}}(x) \stackrel{0}{\subseteq} \chi \Longrightarrow$ $\widehat{\operatorname{Atr}}(x \mid S) \subseteq \chi$.

The relation $\theta_{A t r}$ partition $\mathbb{U}$ into equivalence classes. Let $\widehat{A t r}$ denotes the partition of $\mathbb{U}$, and $\chi$ be any concept in the knowledge system then the lower approximation of $\chi$ is denoted by $\underline{\chi}_{\widehat{A t r}}$ and is defined as

$$
\underline{\chi}_{\widehat{A t r}}=\{x \in U: \widehat{\operatorname{Atr}}(x) \underset{\mathbb{U}}{\stackrel{0}{\complement}} \chi\}
$$

The lower approximation $\underline{\chi}_{\widehat{A t r}}$ is the set of $x \in \mathbb{U}$ which are certainly classified as $\chi$. Another approximation of $\chi$ using $\widehat{A t r}$ is the upper approximation of $\chi$ denoted as $\bar{\chi}_{\widehat{A t r}}$ and is defined as

$$
\bar{\chi}_{\widehat{A t r}}=\{x \in U: \widehat{\operatorname{Atr}}(x) \underset{\mathbb{U}}{\stackrel{\alpha}{\subseteq}} \chi\}, \quad \alpha \in[0,1)
$$

The upper approximation $\bar{\chi}_{\widehat{A t r}}$ is the set of $x \in \mathbb{U}$ which are possibly belongs to $\chi$. The pair $<\bar{\chi}_{\widehat{A t r}}, \underline{\chi}_{\widehat{A t r}}>$ is termed as rough set, and the boundary region $\partial_{\widehat{A t r}}(\bar{\chi})$ of the rough set is given by

$$
\partial_{\widehat{A t r}}(\chi)=\bar{\chi}_{\widehat{A t r}}-\underline{\chi}_{\widehat{A t r}}
$$

The boundary region, also termed as the uncertain-region, is the set of objects we can't classify with certainty using Atr. 


\subsection{Variable precision rough set approxima- tions [8]}

Ziarko [8] generalized the notion of rough set approximations by allowing a specified degree of misclassification error in set approximation. With the inclusion-relation over $U$ and given fix value of misclassification error $\alpha \in[0,0.5)$, the generalized approximations are defined by:

$$
\begin{aligned}
& \chi_{\overline{A t r}}^{\alpha}=\{x \in U: \widehat{\operatorname{Atr}}(x) \underset{\mathbb{U}}{\stackrel{\alpha}{\complement}} \chi\} \\
& \bar{\chi}_{\overline{A t r}}^{\alpha}=\{x \in U: \widehat{\operatorname{Atr}}(x) \underset{\mathbb{U}}{\complement} \chi\}
\end{aligned}
$$

\subsection{Multi-granulation rough set approxima- tions (MGRS) [17]}

Let $(\mathbb{U}, A t r \cup\{D\}, V)$ be the knowledge system, $A t_{1}, A t_{2} \subseteq$ $A t r$, and $\widehat{A t_{1}}, \widehat{A t_{2}}$ be two partitions of $\mathbb{U}$ by the relation $\theta_{A t_{1}}$ and $\theta_{A t_{2}}$ respectively, and $\chi$ be any concept in $\mathbb{U}$, then the approximations of $\chi$ are defined by:

$$
\begin{aligned}
& \chi_{\widehat{A t_{1}}+\widehat{A t_{2}}}=\left\{x \in \mathbb{U}: \widehat{A t_{1}}(x) \stackrel{0}{\stackrel{0}{\complement}} \chi \text { or } \widehat{A t_{2}}(x) \stackrel{0}{\stackrel{0}{\mathbb{U}}} \chi\right\} \\
& \bar{\chi}_{\widehat{A t_{1}}+\widehat{A t_{2}}}=\sim \underline{(\sim \chi)_{\widehat{A t_{1}}}+\widehat{A t_{2}}}
\end{aligned}
$$

\subsection{Multi-granulation variable precision rough set approximations [18]}

Let $\delta$ is the fix predefined value of misclassification error, then the approximations of $\chi$ are given as:

$$
\begin{aligned}
& \underline{\chi}_{\widehat{A t_{1}}+\widehat{A t_{2}}}^{\delta}=\left\{x \in \mathbb{U}: \widehat{A t_{1}}(x) \stackrel{\delta}{\subseteq} \chi \text { or } \widehat{\mathbb{U}} \widehat{A t_{2}}(x) \underset{\mathbb{U}}{\subseteq} \chi\right\} \\
& \bar{\chi} \widehat{A t_{1}}+\widehat{A t_{2}}=\sim \underline{(\sim \chi)} \widehat{\widehat{A t_{1}}+\widehat{A t_{2}}}
\end{aligned}
$$

where $\delta \in[0,0.5)$

\section{Uncertainty optimization model}

This section specifies the multi-granular variable precision rough set in the context of uncertainty optimization. We define the uncertainty optimization based multi-granulation rough set (UOMGRS) approximations, and give its comparisons with the single granulation rough set (SGRS) and multi-granulation rough set (MGRS) approximations.

\subsection{Uncertainty minimization problem}

Let $A t r$ be any $n$-dimensional attribute set, $\delta$ is the specified misclassification error value, and $D$ is the decision attribute. Let $\widehat{D}$ be the partition of $\mathbb{U}$ then each element of $\widehat{D}$ is the concept in the knowledge systeml. Let $\chi_{i} \in \widehat{D}, i=1,2, \ldots, k$ then each $\chi_{i}$ is the concept in the knowledge system. Let $A t_{i} \subseteq A t r, i=1,2, \ldots, m$ denotes the subsets of the attribute set $A t r$, and $\widehat{A t}_{i}(x)$ denotes the equivalence classes of $x \in \mathbb{U}$ by the relation $\theta_{A t_{i}}$ as defined by (1). The positive region $[12,16]$ of the knowledge system with respect to decision attribute $D$ using the attribute set $A t r$ is defined as

$$
\operatorname{POS}_{A t r}(D)=\bigcup_{i=1}^{k}\left\{\underline{\chi_{i} \widehat{A t r}}: \chi_{i} \in \widehat{D}\right\}
$$

The positive region is the set of elements which are classified into the given concept with a certainty. The complement of the positive region is the set of all elements which cannot be classified with a certainty in the given concepts. We call this region as the uncertain region $U n c(A t r)$ of the attribute set Atr i.e.

$$
U n c(A t r)=\sim \operatorname{Pos}_{A t r}(D)
$$

The attribute $A t \subseteq A t r$ for which $U n c(A t)$ is the minimum is the most informative feature subset. Thus we have to minimize the uncertainty region $U n c(A t)$ of the attribute set $A t$ using the positive region $P O S_{A t_{i}}(D)$ of the attribute set $A t_{i}, \quad i=1,2, \ldots, m$. Let $\operatorname{POS}_{(A t \mid U n c(A t))}(D)$ denotes the positive region of $A t_{i}$ in the uncertain region of At then the uncertainty minimization problem is given as:

subject to the constraints

$$
\begin{gathered}
\operatorname{POS}_{\left(A t_{i} \mid \operatorname{Unc}(A t)\right)}(D)=P O S_{A T}(D) \cup\left\{x \in \mathbb{U}: e\left(\widehat{A t}_{i}(x \mid U n c(A t)), \chi_{j}\right) \leq \delta\right\} \\
e\left(\widehat{A t}_{i}(x \mid \mathbb{S}), \chi_{j}\right) \leq \delta, \quad i=1,2, \ldots, n, \quad j=1,2, \ldots, k, \quad \forall S \subseteq \mathbb{U}
\end{gathered}
$$

\subsection{Uncertainty optimization based multi- granulation rough set approximations (UOMGRS)}

Definition 4.2.1: Tolerance error: Let $\widehat{P}(x)$ denotes the class of the equivalence relation $\theta_{P}$ for the attribute set $P$, then we define the tolerance error over $\mathbb{U}$ for the given 
value of misclassification error $\delta$ as the average of the values $e(\widehat{P}(x \mid \mathbb{U}), \chi)$ such that $e(\widehat{P}(x \mid \mathbb{U}), \chi) \leq \delta$.

Let $P$ and $Q$ be two attribute sets, $\chi$ be any concept in the knowledge system, and $\delta$ be the specified value of misclassification error then the lower approximation of $\chi, Q$ given $P$ for the misclassification error value $\delta$ denoted as $\underline{\chi}_{(Q \mid P)}^{\delta}$ and is given by

$$
\underline{\chi}_{\widehat{(Q \mid P)}}^{\delta}=\left\{x \in \mathbb{U}: \widehat{P}(x) \underset{\mathbb{U}}{\stackrel{\alpha}{\subseteq}} \chi \text { or } \widehat{Q}(x) \underset{\sim \underline{\chi}_{\widehat{\mathbb{P}}}^{\alpha}}{\stackrel{\beta}{\subseteq}} \chi\right\}
$$

where $\alpha$ is the tolerance error over $\mathbb{U}$ and $\beta$ is the tolerance error over $\sim \chi_{\widehat{P}}^{\alpha}$ for the misclassification error value $\delta$. Note that $\chi_{\widehat{P}}^{\alpha}$ is the positive region of decision $D$ with respect to the attribute $P$ for the concept $\chi$. Thus $\sim \chi_{\widehat{P}}^{\alpha}$ is the uncertain region of $P$ with respect to the decision class $\chi$. Hence by (20), we minimize the uncertain region of attribute set $P$, using the positive region of attribute set $Q$, for the decision concept $\chi$.

The upper approximation of $\chi, Q$ given $P$ for the misclassification error value $\delta$ denoted as $\bar{\chi} \frac{\delta}{(Q \mid P)}$ and is given by

$$
\bar{\chi}_{\overline{(Q \mid P)}}^{\delta}=\sim(\sim \chi) \frac{\delta}{(Q \mid P)}
$$

It is clear that when $\delta=0$, we have $\alpha, \beta=0$.

Example 3.2.1 Consider a knowledge system ( $U, A t r \cup$ $\{D\}, V)$ containing the information of COVID-19 patients represented in Table 1. It contains the information of eight patients using three attributes. Thus we have $\mathbb{U}=\left\{p_{1}, p_{2}, p_{3}, p_{4}, p_{5}, p_{6}, p_{7}, p_{8}\right\}$, Atr $=$ \{Dry cough, Tireness, Fever $\}$ and the decision attribute $\{D\}=$ Decision, which shows the patient is COVID-19 positive or negative. Each value of the decision attribute give rise to a concept in the knowledge system. Consider a concept $\chi=($ Decision, Positive $)$, then $\chi=\left\{p_{2}, p_{3}, p_{7}, p_{8}\right\}$. Let $P=\{$ Dry Cough $\}$ and $Q=\{$ Tiredness $\}$ are the two sets of attributes, then the classes of the relation $\theta_{P}$ and $\theta_{Q}$ are obtained as follows:

$\widehat{P}=\left\{\left(P 1,\left\{p_{1}, p_{4}, p_{5}\right\}\right),\left(P 2,\left\{p_{2}, p_{3}\right\}\right),\left(P 3,\left\{p_{6}, p_{7}, p_{8}\right\}\right)\right\}$, $\widehat{Q}=\left\{\left(Q 1,\left\{p_{1}, p_{2}, p_{3}, p_{5}, p_{6}\right\}\right),\left(Q 2,\left\{p_{4}, p_{7}\right\}\right),\left(Q 3,\left\{p_{8}\right\}\right)\right\}$

Also, Let $\mathbb{R}=\{P \cup Q\}$ then the classes of the relation $\theta_{R}$ are obtained as:

$\widehat{\mathbb{R}}=\left\{\left(\mathbb{R} 1,\left\{p_{1}, p_{5}\right\}\right),\left(\mathbb{R} 2,\left\{p_{2}, p_{3}\right\}\right),\left(\mathbb{R} 3,\left\{p_{4}\right\}\right),\left(\mathbb{R} 4,\left\{p_{6}\right\}\right)\right.$, $\left.\left(\mathbb{R} 5,\left\{p_{7}\right\}\right),\left(\mathbb{R} 6,\left\{p_{8}\right\}\right)\right\}$.

Let us assume the misclassification error value is assigned as $\delta=0$, thus tolerance error $\alpha, \beta=0$. By definition, we have

$$
\begin{aligned}
\underline{\chi}_{\widehat{P}}^{0} & =\{x \in \mathbb{U}: \widehat{P}(x) \stackrel{0}{\subseteq} \chi\} \\
& =\{x \in \mathbb{U}: e(\widehat{P}(x \mid \mathbb{U}), \chi)=0\} \\
& =\{x \in \mathbb{U}: \widehat{P}(x \mid U) \subseteq \chi\} \\
& =\{P 2\} \\
& =\left\{p_{2}, p_{3}\right\}
\end{aligned}
$$

Therefore, $\sim \underline{\chi}_{\widehat{P}}^{0}=\left\{p_{1}, p_{4}, p_{5}, p_{6}, p_{7}, p_{8}\right\}$. Thus by comput- ing we have,

$$
\begin{aligned}
& \underline{\chi}_{\widehat{(Q \mid P)}}^{0}=\{x \in \mathbb{U}: \widehat{P}(x) \stackrel{0}{\underset{\mathbb{U}}{\subseteq}} \chi \text { or } \widehat{Q}(x) \stackrel{0}{\stackrel{0}{\subseteq}} \chi\} \\
& =\{x \in \mathbb{U}: \widehat{P}(x) \stackrel{0}{\underset{\mathbb{U}}{\subseteq}} \chi\} \cup\{x \in \mathbb{U}: \widehat{Q}(x) \stackrel{0}{\stackrel{0}{\subseteq}} \chi\} \\
& =\left\{p_{2}, p_{3}\right\} \cup\left\{p_{8}\right\} \\
& =\left\{p_{2}, p_{3}, p_{8}\right\}
\end{aligned}
$$

Now, to find upper approximation of $\chi$, we have $\sim \chi=$ (Decision, Negative), i.e. $\sim \chi=\left\{p_{1}, p_{4}, p_{5}, p_{6}\right\}$. We have by definition

$$
\begin{aligned}
& \sim \chi_{P}^{0}=\{x \in \mathbb{U}: \widehat{P}(x) \underset{\mathbb{U}}{\stackrel{0}{\subseteq}} \sim \chi\} \\
& =\left\{p_{1}, p_{4}, p_{5}\right\}
\end{aligned}
$$

Thus, $\sim\left(\underline{\sim}_{\widehat{P}}^{0}\right)=\left\{p_{2}, p_{3}, p_{6}, p_{7}, p_{8}\right\}$. The upper approximation of $\chi, \bar{Q}$ given $P$ is obtained as

$$
\begin{aligned}
& \bar{\chi}_{\overline{(Q \mid P)}}^{0}=\sim(\sim \chi \chi)^{0} \frac{}{(Q \mid P)}
\end{aligned}
$$

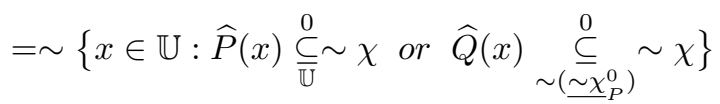

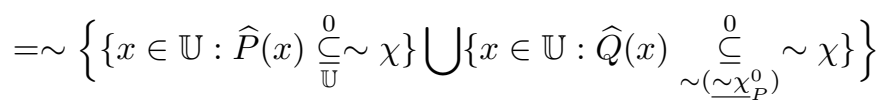

$$
\begin{aligned}
& =\sim\left\{\left\{p_{1}, p_{4}, p_{5}\right\} \cup \phi\right\} \\
& =\left\{p_{2}, p_{3}, p_{6}, p_{7}, p_{8}\right\}
\end{aligned}
$$

Also, the lower and upper approximation of multi-granulation rough set are obtained as:

$$
\begin{aligned}
& \underline{\chi}_{\widehat{P}+\widehat{Q}}=\{x \in \mathbb{U}: \widehat{P}(x) \underset{\mathbb{U}}{\stackrel{0}{\subseteq}} \chi \text { or } \widehat{Q}(x) \underset{\mathbb{U}}{\subseteq} \chi\} \\
& =\left\{\left\{p_{2}, p_{3}\right\} \cup\left\{p_{8}\right\}\right\}=\left\{p_{2}, p_{3}, p_{8}\right\} \\
& \bar{\chi}_{\widehat{P}+\widehat{Q}}=\sim(\sim \chi)_{\widehat{P}+\widehat{Q}}=\sim\left\{\left\{p_{1}, p_{4}, p_{5}\right\} \cup \phi\right\} \\
& =\left\{p_{2}, p_{3}, p_{6}, p_{7}, p_{8}\right\}
\end{aligned}
$$

Also, the Pawlak rough set approximations are obtained as

$$
\begin{aligned}
\underline{\chi}_{\widehat{P \cup Q}} & =\underline{\chi}_{\widehat{R}}=\{x \in \mathbb{U}: \widehat{R}(x) \stackrel{0}{\subseteq} \chi\} \\
& =\left\{p_{2}, p_{3}, p_{7}, p_{8}\right\} \\
\bar{\chi}_{\widehat{P \cup Q}} & =\bar{\chi}_{\widehat{\mathbb{R}}}=\{x \in \mathbb{U}: \widehat{\mathbb{R}}(x) \cap \chi \neq \phi\} \\
& =\left\{p_{2}, p_{3}, p_{7}, p_{8}\right\}
\end{aligned}
$$

The above example illustrates the difference between Pawlak's rough set approximations with multi-granulation rough set approximations, and uncertainty optimization-based rough set approximations. Here we give some of the results regarding various rough set approximations.

Proposition 3.2.1. Let $(\mathbb{U}, A t r \cup\{D\}, V)$ be the knowledge system, $\widehat{P}, \widehat{Q}$ denotes the partition of $\mathbb{U}$ by the relation $\theta_{P}$ and $\theta_{Q}$ respectively and $\chi$ be any concept in the knowledge system. Then we have 
Table 1. Knowledge system containing information of patients

\begin{tabular}{cccccc}
\hline & \multicolumn{3}{c}{ Condition attributes } & & Decision attribute \\
\cline { 2 - 3 } \cline { 5 - 5 } Patient & Dry cough $(P)$ & Tiredness $(Q)$ & Fever $(R)$ & $\{D\}$ \\
\hline$p_{1}$ & Mild & Cumulative & Yes & & Negative \\
$p_{2}$ & Soaring & Cumulative & No & & Positive \\
$p_{3}$ & Soaring & Cumulative & No & & Positive \\
$p_{4}$ & Mild & Transient & Yes & & Negative \\
$p_{5}$ & Mild & Cumulative & No & & Negative \\
$p_{6}$ & No & Cumulative & Yes & & Negative \\
$p_{7}$ & No & Transient & No & \\
$p_{8}$ & No & Circadian & Yes & Positive \\
\hline
\end{tabular}

(1) $\underline{\chi}_{\widehat{P}+\widehat{Q}}^{0} \subseteq \underline{\chi}_{\widehat{(Q \mid P)}}^{0} \subseteq \underline{\chi}_{\widehat{P \cup Q}}$

(2) $\bar{\chi}_{\widehat{P \cup Q}} \subseteq \bar{\chi}_{\widehat{(Q \mid P)}}^{0} \subseteq \bar{\chi}_{\widehat{P}+\widehat{Q}}^{0}$

Proof: If $P=Q(P, Q \subseteq$ Atr $)$, then from (13) we have $\underline{\chi}_{\widehat{P}+\widehat{P}}=\{x \in \mathbb{U}: \widehat{P}(x) \underset{\mathbb{U}}{\stackrel{0}{\complement}} \chi\}=\underline{\chi}_{\widehat{P}}$. Also from (15) we have $\underline{\chi}_{(\overline{P \mid P)}}^{0}=\{x \in \mathbb{U}: \widehat{P}(x) \stackrel{0}{\underset{\mathbb{U}}{C}} \chi\}=\underline{\chi}_{\widehat{P}}(\because$ if $\left.\widehat{P}(x) \underset{\mathbb{U}}{\stackrel{0}{\subset}} \chi \Rightarrow \widehat{P}(x) \underset{\sim \underline{\chi}_{\widehat{P}}^{0}}{\stackrel{0}{\not}} \chi\right)$. Also $\underline{\chi}_{\widehat{P} \cup P}=\underline{\chi}_{\widehat{P}}$. Thus for $P=Q$, we have $\underline{\chi}_{\widehat{P}+\widehat{Q}}^{0}=\underline{\chi}_{(\overline{Q \mid P)}}^{0}=\underline{\chi}_{\widehat{P \cup Q}}$. Similarly, for $P=Q$, we can prove that $\bar{\chi}_{\widehat{P \cup Q}}=\bar{\chi}_{\overline{(Q \mid P)}}^{0}=\bar{\chi}_{\widehat{P}+\widehat{Q}}^{0}$.

Let us suppose that, $P \neq Q$.

(1a) It is clear that if $\widehat{Q}(x) \stackrel{0}{\underset{\mathbb{U}}{\complement}} \chi \Rightarrow \widehat{Q}(x) \stackrel{0}{\stackrel{0}{\subset}} \chi, \forall A \subseteq \mathbb{U}$.

Thus we have

$$
\begin{aligned}
& x \in \underline{\chi}_{\widehat{P}+\widehat{Q}}^{0} \Rightarrow \widehat{P}(x) \stackrel{0}{\underset{\mathbb{U}}{\complement}} \chi \text { or } \widehat{Q}(x) \stackrel{0}{\stackrel{0}{\mathbb{U}}} \chi \\
& \Rightarrow \widehat{P}(x) \underset{\mathbb{U}}{\stackrel{0}{\subseteq}} \chi \text { or } \widehat{Q}(x) \underset{\sim \underline{\chi}_{\bar{P}}^{0}}{\stackrel{0}{\subseteq}} \chi \\
& \Rightarrow \quad x \in \underline{\underline{\chi}} \overline{(\widehat{Q \mid P)}}
\end{aligned}
$$

$$
\therefore \underline{\chi}_{\widehat{P}+\widehat{Q}} \subseteq \underline{\chi}_{(\widehat{Q \mid P)}}
$$

(1b) We have,

$$
\begin{aligned}
x \in \underline{\chi}_{\widehat{(Q \mid P)}} & \Rightarrow \widehat{P}(x) \stackrel{0}{\stackrel{0}{\mathbb{U}}} \chi \text { or } \widehat{Q}(x) \underset{\sim \underline{\chi}_{\widehat{P}}^{0}}{\stackrel{0}{\subseteq}} \chi \\
& \Rightarrow \widehat{P}(x) \cap \widehat{Q}(x) \stackrel{0}{\stackrel{\complement}{\complement}} \chi \\
& \Rightarrow x \in \underline{\chi}_{\widehat{P} \cup Q}
\end{aligned}
$$

$\therefore \underline{\chi}_{\widehat{(Q \mid P)}} \subseteq \underline{\chi}_{\widehat{P \cup Q}}$

(2) From first part we have $\underline{\chi}_{\widehat{P}+\widehat{Q}}^{0} \subseteq \underline{\chi}_{\overline{(Q \mid P)}}^{0} \subseteq \underline{\chi}_{\widehat{P \cup Q}}$. Replacing $\chi$ by $\sim \chi$ we get, $\sim \chi_{\widehat{P}+\widehat{Q}}^{0} \subseteq \underline{\chi}_{(\overline{Q \mid P)}}^{0} \subseteq$ $\simeq \chi_{\widehat{P} \cup Q}$. Taking negation of each term we get $\sim$ $\left(\underline{\sim \chi}_{\widehat{P \cup Q}}\right) \subseteq \sim\left(\underline{\sim}_{\overline{(Q \mid P)}}^{0}\right) \subseteq \sim\left(\underline{\sim}_{\widehat{P}+\widehat{Q}}^{0}\right)$. Thus we have $\bar{\chi}_{\widehat{P} \cup Q} \subseteq \bar{\chi}_{\overline{(Q \mid P)}}^{0} \subseteq \bar{\chi}_{\widehat{P}+\widehat{Q}}^{0}$.
Proposition 3.2.2. Let $(\mathbb{U}, A t r \cup\{D\}, V)$ be the knowledge system, $\widehat{P}, \widehat{Q}$ denotes the partition of $\mathbb{U}$ by the relation $\theta_{P}$ and $\theta_{Q}$ respectively and $\chi$ be any concept in the knowledge system, then we have

(1) $\underline{\chi}_{\widehat{P}}^{0} \cup \underline{\chi}_{\widehat{Q}}^{0} \subseteq \underline{\chi}_{(\overline{Q \mid P)}}^{0}$

(2) $\underline{\chi}_{\widehat{P}+\widehat{Q}}^{0} \subseteq \underline{\chi}_{(\widehat{Q \mid P)}}^{\delta}, \quad \forall \delta \geq 0$

(3) $\bar{\chi}_{\overline{(Q \mid P)}}^{\delta} \subseteq \bar{\chi}_{\widehat{P}+\widehat{Q}}$

\section{Proof:}

(1) Let any $x \in \underline{\chi}_{\widehat{P}}^{0} \Rightarrow \widehat{P}(x) \underset{\widehat{\mathbb{U}}}{\stackrel{0}{C}} \chi$. But $\underline{\chi}_{(\widehat{Q \mid P)}}^{0}=\{x \in$ $\mathbb{U}: \widehat{P}(x) \underset{\mathbb{U}}{\stackrel{0}{\subseteq}} \chi$ or $\left.\underset{\sim \underline{\sim}_{\bar{P}}^{0}}{\stackrel{0}{\subseteq}} \chi\right\}$. Therefore $x \in \underline{\chi}_{(Q \mid P)}^{0}$. Thus $\underline{\chi}_{\widehat{P}}^{0} \subseteq \underline{\chi}_{(\overline{Q \mid P})}^{0}$. Next, we know that if $\widehat{Q}(x) \stackrel{0}{\stackrel{0}{\complement}} \chi \Rightarrow$

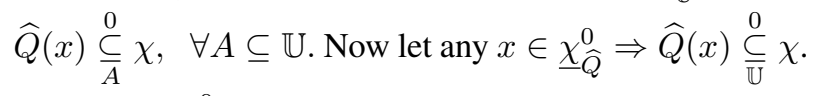
Thus $\widehat{Q}(x) \underset{\sim \underline{\chi}_{\hat{Q}}^{0}}{\stackrel{0}{\subseteq}} \chi\left(\because \sim \underline{\chi}_{\widehat{Q}}^{0} \subseteq \chi\right) . \therefore x \in \underline{\chi}_{\overline{(Q \mid P)}}^{0}$. Thus $\underline{\chi}_{\widehat{Q}}^{0} \subseteq \underline{\chi}_{\overline{(Q \mid P)}}^{0}$. Hence we have $\underline{\chi}_{\widehat{P}}^{0} \cup \underline{\chi}_{\widehat{Q}}^{0} \subseteq \underline{\chi}_{(\bar{Q} \mid P)}^{0}$.

(2) Let any $x \in \underline{\chi}_{\widehat{P}+\widehat{Q}}^{0} \Rightarrow \widehat{P}(x) \underset{\mathbb{U}}{\stackrel{0}{\complement}} \chi$ or $\widehat{Q}(x) \underset{\mathbb{U}}{\stackrel{0}{\subseteq}} \chi$

$\mathbb{U}, 0 \leq \alpha, \beta \leq \delta$

$$
\Rightarrow \widehat{P}(x) \stackrel{\alpha}{\underset{\mathbb{U}}{\subseteq}} \chi \text { or } \widehat{Q}(x) \stackrel{\beta}{\stackrel{\beta}{\perp}} \chi, \forall A \subseteq
$$

$$
\Rightarrow x \in \underline{\chi}_{(\overline{Q \mid P)}}^{\delta}
$$

Thus $\underline{\chi}_{\widehat{P}+\widehat{Q}}^{0} \subseteq \underline{\chi}_{(\overline{Q \mid P)}}^{\delta}$

(3) From part (2), we have $\underline{\chi}_{\widehat{P}+\widehat{Q}}^{0} \subseteq \underline{\chi}_{\overline{(Q \mid P)}}^{\delta}$. Replacing $\chi$ by $\sim \chi$, we get $\sim \chi_{\widehat{P}+\widehat{Q}}^{0} \subseteq \sim \chi_{\widehat{(Q \mid P)}}^{\delta}$. Taking negation of both sides, we get $\sim\left(\underline{\sim \chi}_{\overline{(Q \mid P)}}^{\delta}\right) \subseteq \sim\left(\underline{\sim}_{\widehat{P}+\widehat{Q}}^{0}\right)$. But $\sim(\underline{X})=\bar{X} . \therefore \bar{\chi}_{(\overline{Q \mid P)}}^{\delta} \subseteq \bar{\chi}_{\widehat{P}+\widehat{Q}}$.

Proposition 3.2.3. $\partial_{\widehat{P \cup Q}}(\chi) \subseteq \partial_{\widehat{(Q \mid P)}}(\chi) \subseteq \partial_{\widehat{P}+\widehat{Q}}(\chi)$

Proof: From Pawlak's rough set approximations [12], 
we know that $\underline{\chi}_{\widehat{P \cup Q}} \subseteq \bar{\chi}_{\widehat{P \cup Q}}$. But from Proposition 3.2.1. we have $\underline{\chi}_{\widehat{P}+\widehat{Q}}^{0} \subseteq \underline{\chi}_{\widehat{(Q \mid P)}}^{0} \subseteq \underline{\chi_{\widehat{P \cup Q}}}$ and $\bar{\chi}_{\widehat{P \cup Q}} \subseteq \bar{\chi}_{\widehat{(Q \mid P)}}^{0} \subseteq \bar{\chi}_{\widehat{P}+\widehat{Q}}^{0}$. Thus we have $\underline{\chi}_{\widehat{P}+\widehat{Q}}^{0} \subseteq \underline{\chi}_{\widehat{(Q \mid P)}}^{0} \subseteq \underline{\chi}_{\widehat{P \cup Q}} \subseteq \bar{\chi}_{\widehat{P \cup Q}} \subseteq \bar{\chi}_{\widehat{(Q \mid P)}}^{0} \subseteq \bar{\chi}_{\widehat{P}+\widehat{Q}}^{0}$. $\therefore \bar{\chi}_{\widehat{P \cup Q}}-\underline{\chi}_{\widehat{P \cup Q}} \subseteq \bar{\chi}_{(\widehat{Q \mid P)}}-\underline{\chi}_{\widehat{(Q \mid P)}} \subseteq \bar{\chi}_{\widehat{P}+\widehat{Q}}-\underline{\chi}_{\widehat{P}+\widehat{Q}}$. This implies that $\partial_{\widehat{P \cup Q}}(\chi) \subseteq \partial_{\widehat{(Q \mid P)}}(\chi) \subseteq \partial_{\widehat{P}+\widehat{Q}}(\chi)$.

For the decision attribute $D$, the quality of approximation of $D$ also termed as degree of dependency [2] is given by

$$
\gamma\left(\operatorname{POS}_{A t_{i}}(D)\right)=\frac{\left|\operatorname{POS}_{A t_{i}}(D)\right|}{|\mathbb{U}|}
$$

Example 3.2.2: Let us take knowledge system represented in Table 1. Thus we have $A t r=\{$ Dry cough $(\mathrm{P})$, Tireness $(\mathrm{Q})$, Fever $(\mathrm{R})\}$. Here we are considering each attribute as the singleton attribute subset of Atr. Let us assume that the concept in the knowledge system are denoted by $\chi_{1}=$ (Decision, Positive), and $\chi_{2}=$ (Decision, Negative), then we have $\chi_{1}=\left\{p_{2}, p_{3}, p_{7}, p_{8}\right\}$, and $\chi_{2}=\left\{p_{1}, p_{4}, p_{5}, p_{6}\right\}$. For misclassification error value $\delta=0$, the positive region of the each Singleton attribute set is obtained by using (15) as

$$
\begin{aligned}
& \operatorname{POS}_{P}(D)=\underline{\chi_{1}} \widehat{P} \cup \underline{\chi_{2}} \widehat{Q}=\left\{p_{2}, p_{3}\right\} \cup\left\{p_{1}, p_{4}, p_{5}\right\} \\
& =\left\{p_{1}, p_{2}, p_{3}, p_{4}, p_{5}\right\} \\
& P O S_{Q}(D)=\underline{\chi_{1} \widehat{P}} \cup \underline{\chi_{2}} \widehat{Q}=\left\{p_{8}\right\} \cup \phi=\left\{p_{8}\right\} \\
& P O S_{R}(D)=\underline{\chi_{1}} \widehat{P} \cup \underline{\chi_{2}} \widehat{Q}=\phi \cup \phi=\phi
\end{aligned}
$$

Thus, we have $\left|P O S_{P}(D)\right| \geq\left|P O S_{Q}(D)\right| \geq\left|P O S_{R}(D)\right|$. The positive region of attribute $P$ is the maximum among the attribute subsets of Atr. Hence we choose attribute $P$ as rank 1. Next, by (16), we have uncertain region of the attribute $P$ as $U n c(P)=\left\{p_{6}, p_{7}, p_{8}\right\}$. The positive regions of the remaining attribute subsets in $U n c(P)$ are obtained as

$$
\begin{aligned}
\operatorname{POS}_{(Q \mid U n c(P))}(D) & =\underline{\chi_{1} \widehat{Q}} \cup \underline{\chi_{2} \widehat{Q}}=\left\{p_{7}, p_{8}\right\} \cup\left\{p_{6}\right\} \\
& =\left\{p_{6}, p_{7}, p_{8}\right\} \\
P O S_{(R \mid U n c(P))}(D) & =\underline{\chi_{1} \widehat{R}} \cup \underline{\chi_{2} \widehat{R}}=\left\{p_{7}\right\} \cup \phi=\left\{p_{7}\right\}
\end{aligned}
$$

Thus, we have $\left|P O S_{(Q \mid \operatorname{Unc}(P))}\right| \geq\left|P O S_{(R \mid \operatorname{Unc}(P))}\right|$. Hence we sort attribute $Q$ as rank 2. Now, we have $P O S_{\{P, Q\}}(D)=$ $\mathbb{U}$, and by (22), $\gamma\left(P O S_{\{P, Q\}}(D)\right)=1$. Hence the sorted attribute list of Atr according to rank is $\{P, Q\}$.

Here we give an algorithm to find the given attribute set's positive region and sort them according to their positive region for data reduction. Initially, we consider each attribute as a singleton subset of the given attribute set and finds each attribute's positive region. Let $\operatorname{Pos}(F)$ denotes the positive region of the feature set $F \subseteq A t r$ with respect to the decision attribute $D$. Next, we minimize the attribute's uncertain-region, whose positive region is the maximum using the remaining attributes' positive regions. The Algorithm 1 find and sort attributes according to their positive region. We measure the attribute's quality of approximation to group them for uncertainty minimization, using the proposed uncertainty minimization problem. We have used three data-sets from UCI machine learning
[19] in our study. The information of the used data-sets is provided in Table 2 .

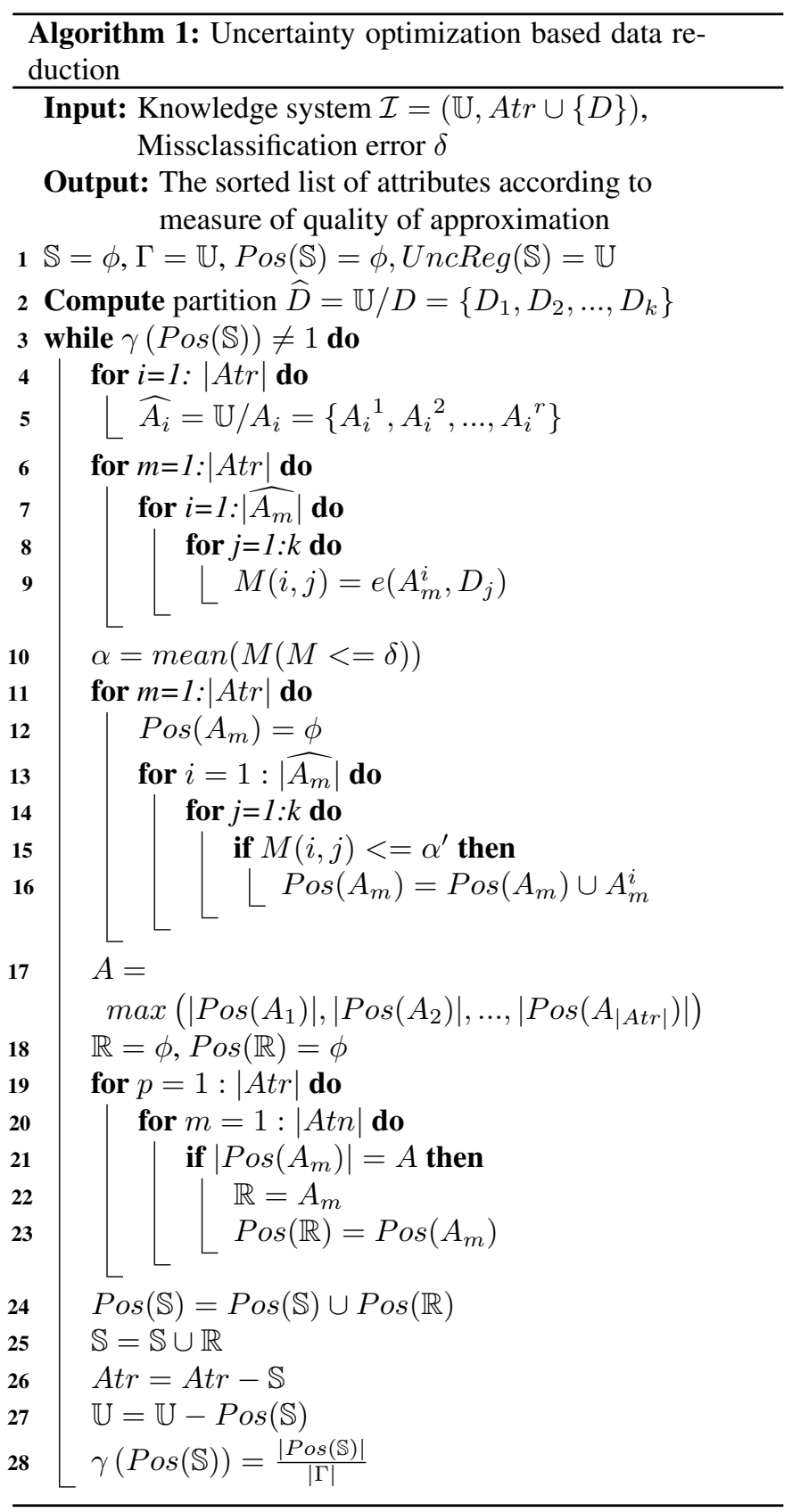

Table 2. Information of the data-sets used in the study

\begin{tabular}{cccc}
\hline Data-set & Instances & Attributes & Concepts \\
\hline Tic-Tac-Toe & 958 & 9 & 2 \\
Dermatology & 366 & 33 & 6 \\
Car & 1728 & 6 & 4 \\
\hline
\end{tabular}

Here we give the step-by-step information about the selected attributes using their quality of approximation by the proposed algorithm. We assigned the misclassification error value as $\delta=0.26$ because we had an empty positive region of all the attributes for less than $\delta=0.26$. Here we denote $\gamma\left(P O S_{A t_{i}}(D)\right.$ 
Table 3. Iteration wise sorted attributes list according to quality of approximation for misclassification error value $\delta=0.26$

\begin{tabular}{|c|c|c|c|c|c|c|c|c|c|c|c|c|}
\hline & Region & $\gamma\left(A_{1}\right)$ & $\gamma\left(A_{2}\right)$ & $\gamma\left(A_{3}\right)$ & $\gamma\left(A_{4}\right)$ & $\gamma\left(A_{5}\right)$ & $\gamma\left(A_{6}\right)$ & $\gamma\left(A_{7}\right)$ & $\gamma\left(A_{8}\right)$ & $\gamma\left(A_{9}\right)$ & $\overline{A T T}$ & $\gamma(A T T)$ \\
\hline Iter 1 & $\mathbb{U}$ & 0 & 0 & 0 & 0 & 0.214 & 0 & 0 & 0 & 0 & $\left\{A_{5}\right\}$ & 0.214 \\
\hline Iter 2 & $U n c(A T T)$ & 0.387 & 0.134 & 0.048 & 0.090 & - & 0.071 & 0 & 0 & 0.113 & $\left\{A_{5}, A_{1}\right\}$ & 0.520 \\
\hline Iter 3 & $U n c(A T T)$ & - & 0.078 & 0.035 & 0 & - & 0.039 & 0.102 & 0.035 & 0.177 & $\left\{A_{5}, A_{1}, A_{9}\right\}$ & 0.697 \\
\hline Iter 4 & $U n c(A T T)$ & - & 0 & 0.139 & 0 & - & 0.081 & 0.058 & 0.046 & - & $\left\{A_{5}, A_{1}, A_{9}, A_{3}\right\}$ & 0.836 \\
\hline Iter 5 & $U n c(A T T)$ & - & 0 & - & 0.045 & - & 0.023 & 0.091 & 0 & - & $\left\{A_{5}, A_{1}, A_{9}, A_{3}, A_{7}\right\}$ & 0.927 \\
\hline Iter 6 & $U n c(A T T)$ & - & 0 & - & 0 & - & 0 & - & 0.043 & - & $\left\{A_{5}, A_{1}, A_{9}, A_{3}, A_{7}, A_{8}\right\}$ & 0.970 \\
\hline Iter 7 & $U n c(A T T)$ & - & 0.030 & - & 0 & - & & 0 & - & - & $\left\{A_{5}, A_{1}, A_{9}, A_{3}, A_{7}, A_{8}, A_{2}\right\}$ & 1 \\
\hline
\end{tabular}

Table 4. Quality of approximation with different number of attributes in data-set Tic-Tac-Toe

\begin{tabular}{|c|c|c|c|c|c|c|c|c|c|}
\hline \multirow[t]{2}{*}{ Measure } & \multicolumn{9}{|c|}{ Attributes } \\
\hline & 1 & 2 & 3 & 4 & 5 & 6 & 7 & 8 & 9 \\
\hline$\gamma$ in SGRS [17] & 0 & 0 & 0.138 & 0.165 & 0.423 & 0.788 & 0.946 & 1.00 & 1.00 \\
\hline$\gamma$ in MGRS [17] & 0 & 0 & 0 & 0 & 0 & 0 & 0 & 0 & 0 \\
\hline$\gamma$ in UOMGRS & 0.212 & 0.524 & 0.701 & 0.849 & 0.936 & 0.977 & 1.00 & 1.00 & 1.00 \\
\hline
\end{tabular}

Table 5. Quality of approximation with different number of attributes in data-set Dermatology

\begin{tabular}{|c|c|c|c|c|c|c|c|c|c|c|c|}
\hline \multirow[t]{2}{*}{ Measure } & \multicolumn{11}{|c|}{ Attributes } \\
\hline & 3 & 6 & 9 & 12 & 15 & 18 & 21 & 24 & 27 & 30 & 33 \\
\hline$\gamma$ in SGRS [17] & 0.044 & 0.607 & 0.855 & 0.897 & 0.981 & 0.995 & 1.000 & 1.000 & 1.000 & 1.000 & 1.000 \\
\hline$\gamma$ in MGRS [17] & 0 & 0 & 0 & 0 & 0 & 0 & 0 & 0 & 0 & 0 & 0 \\
\hline$\gamma$ in UOMGRS & 0.044 & 0.642 & 0.907 & 0.9856 & 0.994 & 1.000 & 1.000 & 1.000 & 1.000 & 1.000 & 1.000 \\
\hline
\end{tabular}

Table 6. Quality of approximation with different number of attributes in data-set Car

\begin{tabular}{lcccccc}
\hline Measure & Attributes & & & & & \\
\cline { 2 - 7 } & 1 & 2 & 3 & 4 & 5 & 6 \\
\hline$\gamma$ in SGRS [17] & 0.000 & 0.187 & 0.188 & 0.458 & 0.481 & 1.000 \\
$\gamma$ in MGRS [17] & 0.000 & 0.000 & 0.000 & 0.333 & 0.333 & 0.333 \\
$\gamma$ in UOMGRS & 0 & 0.455 & 0.515 & 0.633 & 0.857 & 1.000 \\
\hline
\end{tabular}

by $\gamma\left(A t_{i}\right)$. Also, let $A T T$ denotes an iteration-wise sorted list of attributes. The iteration-wise chosen attribute is given in Table 3.

From Table 3, we have the sorted list of attributes for the Tic-Tac-Toe data-set according to their quality of approximation as $\left\{A_{5}, A_{1}, A_{9}, A_{3}, A_{7}, A_{8}, A_{2}\right\}$. Here, we have sorted the attribute according to the positive region. The most informative attribute is the one whose positive region is the maximum. Hence we minimize the uncertain region of the the most informative attribute using the positive region of the remaining attributes. Thus by using uncertainty optimization based multi-granulation rough set, we get greater measure of quality of approximation as compared to other approaches of approximation. We have compared the quality of approximation of the attribute set by UOMGRS with that of SGRS and MGRS. The quality of approximations of the attributes with different approaches for data-sets is listed in Table 4-6 and Figure 1-3. The comparison shows that, the quality of approximation with UOMGRS is greater than that of SGRS and MGRS.

The SGRS requires 8 out of 9 attributes for approximation of the concepts in the Tic-Tac-Toe dataset. Also, SGRS required 21 attributes out of 33 for approximation of the Dermatology dataset concepts and required 6 out of 6 attributes for Car dataset concepts. Thus it required 35 out of 48 attributes to approximate the concepts in the three datasets, i.e., approximately $72 \%$ of the attributes. Also, MGRS requires 48 out of

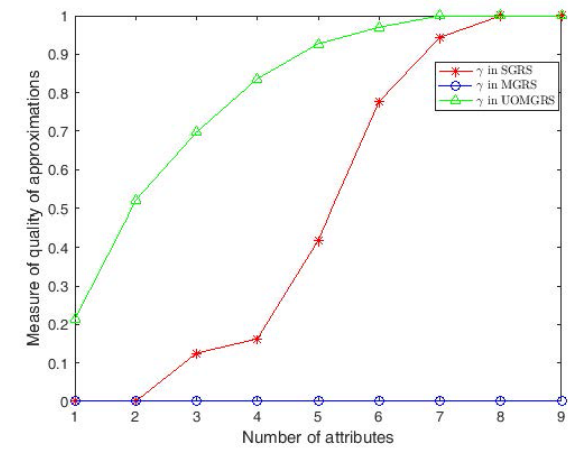

Figure 1. Variation of quality of approximations against number of attributes (Tic-Tac-Toe data-set)

48 attributes for approximation of concepts in the dataset, i.e., it requires $100 \%$ attributes to approximate the concepts in the datasets. In comparison, UOMGRS requires 30 out of 48 attributes in the three data sets to approximate the concepts, i.e., approximately $62 \%$ attributes.

\section{Discussion and Conclusions}

In this work, we have extended the multi-granulation variable precision rough set in the context of uncertainty optimization. As a result, we formulated the uncertainty optimization- 


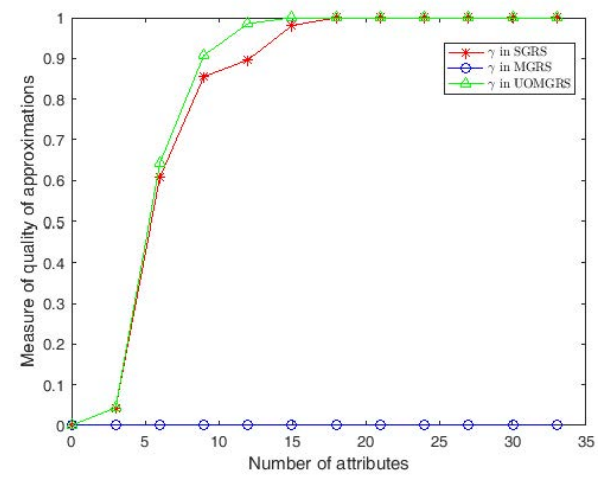

Figure 2. Variation of quality of approximations against number of attributes (Dermatology data-set)

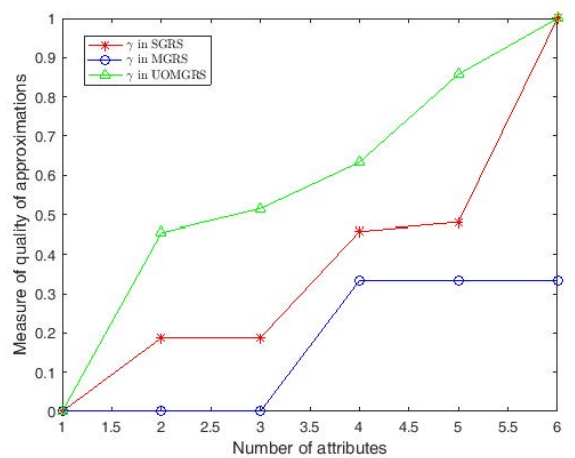

Figure 3. Variation of quality of approximations against number of attributes (Car data-set)

based multi-granulation rough set approximation (UOMGRS). Using the proposed method, we find the attribute with the highest quality of the approximation as it is the most informative attribute in the feature space. It is desirable to minimize the rough set boundary region using the attribute having the highest approximation quality. Thus we group the attributes whose relative quality of approximation is the maximum to maximize the positive region and to minimize the uncertain region. The subsets whose quality of the approximation is $100 \%$ are the reducts of the attribute set. An algorithm based on uncertainty optimization is given for sorting the attributes according to their approximation quality and feature grouping to maximize the quality of approximation. The UOMGRS is compared with the single granulation rough set (SGRS) and multi-granulation rough set (MGRS). The measure of the quality of approximation using UOMGRS is found more remarkable than that of SGRS and MGRS. The SGRS and MGRS required an average of at least $72 \%$ attributes for approximation of concepts in the dataset. In comparison, UOMGRS only needed an average of $62 \%$ of the attributes for approximation of the concepts in the dataset. So by our proposed method, object classification is possible with comparatively less dataset. The method helps minimize the uncertainties in the dataset in a more efficient way. The study provides a novel and more effective approach for minimizing boundary regions and its application for data reduction.

\section{Acknowledgements}

We are thankful to National Institute of Technology Raipur for providing space and facilities for work.

\section{Conflict of Interest}

The authors declare that there is no conflict of interest.

\section{REFERENCES}

[1] D'eer, L., Cornelis, C., and Yao, Y., "A semantically sound approach to pawlak rough sets and covering-based rough sets", International Journal of Approximate Reasoning, 78, 62-72, 2016

[2] Pawlak, Z., "Rough sets: Theoretical aspects of reasoning about data", vol. 9. Springer Science \& Business Media, 2012

[3] Das, A. K., Sengupta, S., and Bhattacharyya, S., "A group incremental feature selection for classification using rough set theory based genetic algorithm", Applied Soft Computing, 65 , 400-411, 2018

[4] Lei, L., "Wavelet neural network prediction method of stock price trend based on rough set attribute reduction", Applied Soft Computing, 62, 923-932, 2018

[5] Luo, C., Li, T., Chen, H., Fujita, H., and Yi, Z., "Incremental rough set approach for hierarchical multicriteria classification", Information Sciences, 429, 72-87, 2018

[6] Qian, Y., Liang, X., Wang, Q., Liang, J., Liu, B., Skowron, A., Yao, Y., Ma, J., \& Dang, C., "Local rough set: a solution to rough data analysis in big data", International. Journal of Approximate Reasoning, 97 , 38-63, 2018

[7] Sharma, H. K., Kumari, K., and Kar, S., "A rough set approach for forecasting models. Decision Making: Applications in Management and Engineering", 3 (1), 1-21, 2020

[8] Ziarko, W., "Variable precision rough set model" Journal of computer and system sciences, 46 (1), 39-59, 1993

[9] Yasnitsky, L., Gladkiy, S., "New possibilities of application of artificial intelligence methods for high-precision solution of boundary value problems", Mathematics and Statistics Vol. 8(3), pp. 323 - 333

[10] Patricia, A., and Inma, A., "From Exploratory Data Analysis to Exploratory Spatial Data Analysis", Mathematics and Statistics 8 (2): $82-86,2020$

[11] Wang, C., Shao, M., He, Q., Qian, Y., and Qi, Y., "Feature subset selection based on fuzzy neighborhood rough sets", Knowledge-Based Systems, 111, 173-179, 2016 
[12] Huang, Y., Li, T., Luo, C., Fujita, H., and Horng, S. J., ”Dynamic variable precision rough set approach for probabilistic set-valued information systems" Knowledge-based systems, $122,131-147,2017$

[13] Kang, X., and Miao, D., "A variable precision rough set model based on the granularity of tolerance relation", KnowledgeBased Systems, 102 , 103-115, 2016

[14] Jiang, H., Zhan, J., and Chen, D., "Covering-based variable precision (I; T)-fuzzy rough sets with applications to multiattribute decision-making", IEEE Transactions on Fuzzy Systems, 27 (8), 1558-1572, 2016

[15] Qian, Y., Liang, X., Wang, Q., Liang, J., Liu, B., Skowron, A., Yao, Y., Ma, J., and Dang, C., "Local rough set: a solution to rough data analysis in big data", International Journal of Approximate Reasoning, 97 , 38-63, 2018
[16] Wei, W., Liang, J., Qian, Y., and Wang, F., "Variable precision multi-granulation rough set" In 2012 IEEE International Conference on Granular Computing, (pp. 536-540). IEEE, 2012

[17] Qian, Y., Liang, J., Yao, Y., and Dang, C., "Mgrs: A multigranulation rough set", Information sciences, 180 (6), 949-970, 2010

[18] Yang, X., Xu, S., Dou, H., Song, X., Yu, H., \& Yang, J., "Multigranulation rough set: a multiset based strategy", International Journal of Computational Intelligence Systems, 10 (1), 277-292, 2017

[19] UCI machine learning repository, https://archive.ics.uci.edu/ml/datasets.php 